\title{
Circular RNAs: a new frontier for cancer diagnosis and therapy
}

\author{
Miaoci Zhang and Yan Xin ${ }^{*}$
}

\begin{abstract}
In recent years, circular RNAs (circRNAs) have attracted considerable attention because they play a significant role in many fields of cancer biology. Additionally, it has become increasingly clear that circRNAs have the potential to make contributions to the successful application of individualized cancer medicine. This brief review introduces circRNAs by describing their potential as a biomarker and therapeutic target and discussing the possible strategies to target them. This review also presents the challenges that are encountered by circRNAs for their definitive entry into clinical practice. Clearly, our understanding of circRNAs helps to add a new dimension to the molecular structure of cancer and will provide many new opportunities for cancer treatment.
\end{abstract}

Keywords: CircRNA, Cancer, Biomarker, Therapy, Diagnosis, Prognosis

\section{Background}

In the last decade, the extensive application of new innovative technologies has promoted the fast development of many aspects of the RNA field [1]. In particular, nextgeneration sequencing has helped to realize the discovery of circular RNA (circRNAs) and bring circRNAs to the center stage in cancer genomics research [2,3]. As a new type of RNA, circRNAs are expressed endogenously as covalently closed, single-stranded circular molecules [4]. Most circRNAs are the result of a "back-splice" reaction, during which an upstream acceptor splicing site is joined with a donor splicing site $[5,6]$. Originating as exons, introns, or both, circRNAs fall into three categories [7]. Exonic circRNAs (ecRNAs) originate in the sequence of pre-mRNAs during the process of exon skipping. Reverse-oriented repeats that usually appear in the introns on both sides of the exons in ecRNAs can be important for the circularization of exons [8]. Intronic circRNAs (ciRNAs) that consist of one or more introns are derived from linear RNA. Specific 11-nt C-rich element near the branch-point site and 7-nt GU-rich motifs near the $5^{\prime}$ splice site are vital for the biosynthesis of ciRNA [9]. Exon-intron circRNAs (ElciRNAs) are another type of circRNA stemming from both introns and exons. The circularization of EIciRNAs can be improved by the

\footnotetext{
*Correspondence: yxin@cmu.edu.cn; yxincmu@163.com

Laboratory of Gastrointestinal Onco-Pathology, Cancer Institute \& General Surgery Institute, The First Affiliated Hospital of China Medical University, 155 Nanjing North Street, Heping District, Shenyang, Liaoning Province 110001, China
} cRNAs, two are located in the nucleus (ciRNAs and EIciRNA) and one is cytoplasmic (ecRNA) [11]. Most genes with circular isoforms produce only one or two distinct circRNAs, although some produce tens of distinct circular products [12]. Although the mechanism of circRNA biogenesis cannot be understood completely, the complementary sequence in the flanking introns is the basis of the biogenesis for the circRNAs of many mammals [5, 13]. Additionally, some activating or antagonistic trans-acting factors, such as quaking and ADAR, can modulate the expression of mammalian circRNAs [14, 15]. Many scientific studies demonstrated numerous potential functions of cirAs that clarify how circRNA changes from negative to its current popular rank [16]. Most circRNAs are 作 express in the saiv, blood, and them promising biomarkers for diagnosis, prognosis, and therapeutic response for cancer patients [18]. Furthermore, in recent years, several studies have indicated that circRNAs exhibited a powerful functional potential in regulating proliferation, apoptosis, angiogenesis, and metastasis, suggesting that circRNAs may be important target molecules in cancer $[19,20]$. This review briefly introduces what is currently known about the potential of circRNA as a biomarker and therapeutic target in cancer and discusses their application prospects in the clinical field. 


\section{Biological functions of circRNAs}

\section{CircRNAs as microRNA and protein sponges}

As a new type of competing endogenous RNA (ceRNA) that is widely believed to decoy relevant microRNAs (miRNAs), circRNAs can impact the bio-functions of miRNAs and thus play an important role in the regulation of miRNA target genes. Compared with other ceRNAs, circRNA has a far better ability to bind miRNAs; thus, it is also called a "super sponge". This is best illustrated with the example of circular RNA sponge for miR-7 (ciRS-7), which sponges miR-7 efficiently with over 70 miR-7 binding sites [21, 22]. A high level of endogenous interactivity between them could be observed in the mouse brain, especially in the hippocampal and neocortical neurons [21]. Ectopic expression of human ciRS-7 mimicked the phenotype caused by knockdown of miR-7, indicating that ciRS-7 can act as a sponge [22]. Having a strong resistance to instability mediated by miRNA, it becomes a useful inhibitor of miR-7 activities, and the transcript levels of miR-7 targets are increased [21, 22]. Another RNA circle, originating at sexdetermining region $\mathrm{Y}$ (SRY), is composed of 16 binding sites of miR-138, and it functions as the sponge of miR-138, thereby upregulating the expression of miR-138 target genes [21, 23].

Another new function of circRNA is that it can serve as a protein decoy which harbors binding sites for a single or multiple proteins and thus regulate gene expression. For example, both Argonaute (AGO) proteins and RNA polymerase II (Pol II) are correlated with circRNA [9, 24]. As "scaffolding" for RNA-binding proteins, circRNAs can facilitate steady interaction and bind multiple proteins because of their intrinsic stability. For instance, circFoxo3 binds to p21 and CDK2, leading to the formation of the ternary complex circFoxo3-p21-CDK2. Therefore, both the biological effect of CDK2 and the progression of the cell cycle can be arrested [25]. Another supported example is circMBL which sequesters MBL protein and prevents it from binding to other targets [5].

\section{CircRNAs as transcriptional regulators}

Recent advances have revealed that circRNAs can play a transcriptional regulatory role. Located in the nucleus, ciRNAs in human cells can regulate the transcription of parent genes in cis. Certain ciRNAs, such as ci-sirt7, ci-mcm5, and ci-ankrd52, interact with the extended RNA Pol II complex. The transcription of sirt7, mcm5, and ankrd52 genes can be reduced via the depletion of ciRNAs, showing that the Pol II transcription of parent genes can be greatly enhanced through nuclear-enriched ciRNAs [9]. Additionally, accumulating evidence has revealed that EIciRNAs, such as EIciPAIP2 and EIciEIF3J, can interact with the U1 small nuclear ribonucleoprotein (snRNP) and RNA Pol II in the promoter region to realize the better transcription of the parent gene. Each EIciRNA contains one U1 small nuclear
RNA (snRNA)-binding site at the retained introns. If the RNA-RNA interaction were blocked, the combination between Pol II and EIciRNA would be reduced, and fewer EIciRNA-U1 snRNP complexes would be bound to the coding gene promoter. Thus, the transcription of genes could be decreased [10].

\section{CircRNAs can be translated}

Convincing evidence has shown that circRNAs can encode for proteins. When an internal ribosome entry site (IRES) is engineered in, circRNAs can be translated in vitro and in vivo effectively, showing that circRNAs can participate in translation [26, 27]. In addition, the translation of circRNA in the cells of human bodies can be realized merely through rolling circle amplification (RCA) without translational element [28]. Weingarten-Gabbay et al. conducted a survey revealing that massive circRNAs have a novel capindependent translation mechanism [29]. Meanwhile, Legnini et al. and Pamudurti et al. also provided in vitro and in vivo evidence to prove that cap-independent translation exists in circRNAs [30, 31]. Additionally, Yang et al. found that human cells are pervasive with circRNA translation driven by $N^{6}$-methyladenosine (m6A) [32].

\section{CircRNAs as potential biomarkers in cancer}

CircRNAs are not sensitive to ribonucleases, such as exonuclease or RNase R, and have a longer half-life with a more prominent specificity in histology. Meanwhile, compared with detecting proteins through antigenantibody reactions, testing circRNAs through quantitative real-time PCR (qRT-PCR) and in situ hybridization are more specific and sensitive. Herein, circRNAs are potential biomarkers in cancer [33].

\section{CircRNAs in cancer diagnosis}

It is well known that most cancer types can be cured, if diagnosed at an early stage. Commonly used cancer diagnostic markers and tools include magnetic resonance imaging, computerized tomographic scanning, and histopathology. However, some of these methods are invasive and expensive. Therefore, minimally invasive and inexpensive methods are required. The fact that circRNAs are highly dysregulated in several cancer types and exhibit a high degree of tissue- and disease-specificity make them an ideal candidate for cancer diagnosis (Table 1). For example, hsa_circ_0013958 was demonstrated to be significantly upregulated in the tissue and plasma of lung adenocarcinoma (LAC). Furthermore, its levels were correlated with lymphatic metastasis and tumor-nodemetastasis (TNM) stage and the area under the receiver operating characteristic (ROC) curve was 0.815 , suggesting that it could be a promising biomarker for LAC [34]. In another study, Li et al. found that 257 new circRNA species were detected and 67 circRNAs were missing in 
Table $1 \mathrm{~A}$ list of circRNAs with potential in cancer diagnosis

\begin{tabular}{|c|c|c|c|c|}
\hline Cancer type & CircRNA & Expression & Source & Reference \\
\hline \multirow[t]{2}{*}{ Bladder cancer } & CircMYLK & Up & Tissue & {$[52]$} \\
\hline & CircTCF25 & Up & Tissue & [85] \\
\hline Prostate cancer & CircSMARCA5 & Up & Tissue & {$[86]$} \\
\hline Glioma & CircTTBK2 & Up & Tissue & [87] \\
\hline Osteosarcoma & Circ_0001564 & Up & Tissue & {$[88]$} \\
\hline \multirow[t]{3}{*}{ Breast cancer } & Circ_0001982 & Up & Tissue & [89] \\
\hline & $\begin{array}{l}\text { Circ_103110, } \\
\text { circ_104689 } \\
\text { and circ_104821 }\end{array}$ & Up & Tissue & [90] \\
\hline & CircABCB10 & Up & Tissue & [91] \\
\hline \multirow[t]{5}{*}{ Hepatocellular cancer } & CiRS-7 & Up & Tissue & [92] \\
\hline & CircZFR & Up & Tissue & [93] \\
\hline & CircFUT8 & Up & Tissue & [93] \\
\hline & CirclPO11 & Up & Tissue & [93] \\
\hline & Circ_0005075 & Up & Tissue & [94] \\
\hline Esophageal cancer & Circ_0067934 & Up & Tissue & [95] \\
\hline \multirow[t]{3}{*}{ Colorectal cancer } & CircBANP & Up & Tissue & [96] \\
\hline & Circ_0020397 & Up & Tissue & [97] \\
\hline & CircKLDHC10 & Up & Serum & [35] \\
\hline Lung adenocarcinoma & Circ_0013958 & Up & Serum & [34] \\
\hline
\end{tabular}

colorectal cancer (CRC) patients compared with those in healthy controls based on serum exosome RNA sequencing (RNA-seq) datasets. Furthermore, it was confirmed that upregulation of serum circ-KLDHC10 was able to distinguish CRC patients from healthy subjects, suggesting that it is a promising non-invasive biomarker for the early detection and screening of CRC [35].

CircRNAs can also help in risk assessment of cancer susceptibility. Single nucleotide polymorphism (SNP) refers to a difference in a single DNA building block of a gene (nucleotide) or within the regulatory regions of a gene. SNPs can be used as a biomarker for predicting an individual's risk of cancer [36]. A study from China consisting of 1600 ethnic Han patients with hepatocellular cancer (HCC) and 1800 cancer-free controls, six tagSNPs were identified in circITCH: rs6059851, rs6120663, rs10485505, rs4911154, rs7266300, and rs11167234. Consequently, rs10485505 and rs4911154 were significantly associated with the increased risk of HCC [37]. This study clearly indicates an association of circRNA SNPs with cancer susceptibility. However, how circRNA SNPs contribute to cancer pathogenesis is not properly defined. The larger, welldesigned in-depth studies from different ethnic populations will validate contributions of circRNA SNPs in cancer risk assessment.
The above examples are only a fraction of the several instances in which circRNAs have demonstrated promising diagnostic potential. More studies are required before circRNAs can be recommended for human use.

\section{CircRNAs in cancer prognosis}

Prognostic evaluation is of great significance for the early intervention of poor prognostic factors and the prolongation of the life expectancy of cancer patients. Recently, a great deal of research showed that examination of circRNA may be useful in the prognostic prediction of cancer (Table 2). For instance, glioblastoma patients with higher circ-FBXW7 had an increased overall survival (OS) compared with those with low expression [38]. In addition, it was found that upregulated circUBAP2 in osteosarcoma tissues was significantly associated with a lower survival rate [39]. Furthermore, circ_100876 upregulation was closely related to shorter OS, lymphatic metastasis and tumor staging in nonsmall cell lung cancer patients [40].

The currently available cancer drugs cannot eradicate cancer cells completely, and most cancer types frequently relapse over a period of time. Therefore, biomarkers that can predict tumor recurrence could be of prognostic significance. Recently, several studies showed that circRNAs could be utilized as potential biomarkers for the prediction of tumor recurrence. For example, the expression of hsa_ circ_0058246 was statistically significantly increased in patients who suffered from the recurrence of gastric cancer (GC) [41]. In another study, patients with lower levels of circPVT1 in GC had a significantly shorter progression-free survival (PFS) than those with high levels of circPVT1, indicating that circPVT1 might be a biomarker for tumor recurrence [42]. Additionally, circBRAF could also be a candidate biomarker for predicting glioma recurrence [43]. Hence, circRNAs have shown potential as biomarkers for predicting recurrence in several cancer types, which could help the clinicians to manage disease before recurrence.

In conclusion, both upregulation and downregulation of circRNA expression could be of prognostic significance. We believe that the use of multiple sensitive and reliable detection methods in large-scale, multi-center studies will help to unravel the true prognostic significance of circRNAs in cancer patients.

\section{CircRNAs in predicting response to chemoradiotherapy}

Recent studies have found that circRNAs impact the sensitivity of tumors to chemoradiotherapy. For instance, $\mathrm{Su}$ et al. revealed that circ_001059, circ_100385 and circ_104983 had the largest upregulation; circ_000695, circ_101877, and circ_102913 had the largest downregulation in radioresistant oesophageal cancer cells according to the microarray data, indicating that these 
Table 2 A list of circRNAs with potential in cancer prognosis

\begin{tabular}{|c|c|c|c|c|c|}
\hline Cancer type & CircRNA & Expression & Source & Association & Reference \\
\hline Glioblastoma & CircFBXW7 & Down & Tissue & OS & [38] \\
\hline Osteosarcoma & CircUBAP2 & Up & Tissue & TNM and OS & [39] \\
\hline \multirow[t]{2}{*}{ Lung cancer } & Circ_100876 & Up & Tissue & Lymph node metastasis,TNM and OS & [40] \\
\hline & CirclTCH & Down & Tissue & TNM & [53] \\
\hline \multirow[t]{2}{*}{ Laryngeal cancer } & Circ_100855 & Up & Tissue & Lymph node metastasis and TNM & [98] \\
\hline & Circ_104912 & Down & Tissue & Lymph node metastasis and TNM & [98] \\
\hline Bladder cancer & CircHIPK3 & Down & Tissue & Vascular invasion and lymph node metastasis & [99] \\
\hline \multirow[t]{5}{*}{ Colorectal cancer } & CircCCDC66 & Up & Tissue & OS & [59] \\
\hline & Circ_001569 & Up & Tissue & Distant metastasis, differentiation, and TNM & [100] \\
\hline & Circ_103809 & Down & Tissue & TNM & [101] \\
\hline & Circ_104700 & Down & Tissue & Distal metastasis & [101] \\
\hline & CiRS-7 & Up & Tissue & Lymph node, distant metastasis, and OS & [51] \\
\hline \multirow[t]{4}{*}{ Hepatocellular cancer } & Circ_0003570 & Down & Tissue & Metastasis & [102] \\
\hline & CircMTO1 & Down & Tissue & OS & [103] \\
\hline & Circ_100338 & Up & Tissue & $\begin{array}{l}\text { Cumulative survival rate, lung metastasis, } \\
\text { vascular invasion, and TNM }\end{array}$ & [104] \\
\hline & CircZKSCAN1 & Down & Tissue & Microscopic vascular invasion and TNM & [60] \\
\hline \multirow[t]{10}{*}{ Gastric cancer } & Circ_104916 & Down & Tissue & TNM and lymph node metastasis & [105] \\
\hline & Circ_0006633 & Down & Tissue & Distal metastasis & [106] \\
\hline & Circ_0000096 & Down & Tissue & TNM & [61] \\
\hline & Circ_0000181 & Down & Tissues and plasma & Lymphatic metastasis and distal metastasis & [107] \\
\hline & Circ_100269 & Down & Tissue & OS & [108] \\
\hline & Circ_002059 & Down & Tissues and plasma & TNM and metastasis & [109] \\
\hline & Circ_0000190 & Down & Tissue & TNM, lymphatic metastasis, and distal metastasis & [110] \\
\hline & CiRS-7 & Up & Tissue & OS & [50] \\
\hline & Circ_0014717 & Down & Tissue & Distal metastasis and TNM & [111] \\
\hline & Circ_0003159 & Down & Tissue & Distal metastasis and TNM & [112] \\
\hline
\end{tabular}

dysregulated circRNAs may be involved in the development of radiation resistance [44]. Another recent study showed that certain fusion-circRNAs suggest resistance to apoptosis-inducing chemotherapy, such as f-circM9, which confers protection to leukemic cells upon treatment with arsenic trioxide [45]. Additionally, Xiong et al. performed microarray analysis of 5-fluorouracil (5FU) chemoradiation-resistant CRC cells and identified hsa_circ_0007031, hsa_circ_0000504, and hsa_circ 0007006 that are the top three upregulated circRNAs among differentially expressed circRNAs, indicating that they are potential biomarkers to predict chemoradiation resistance in CRC [46]. A study conducted by $\mathrm{Gao}$ et al. revealed that the expression of hsa_circ_00006528 in adriamycin (ADM)-resistant human breast cancer tissues and cell lines were obviously higher than that in ADM-sensitive groups, suggesting the potential of circ_0006528 in predicting chemotherapy resistance in breast cancer [47].
In conclusion, circRNAs as biomarkers predicting chemoradiotherapy response in individual patients are essential for improving the clinical management of cancer.

\section{Therapeutic potential of circRNAs in cancer CircRNA as potential therapeutic targets}

An increasing amount of evidence has demonstrated the relationship between various novel circRNAs and signaling pathways with carcinogenesis or with the manipulation of aggressive characteristics of cancer cells, both of which are putative therapeutic targets for novel drugs to increase the survival of cancer patients $[48,49]$. One example is ciRS-7, which was significantly upregulated in GC tissues compared with matched para-carcinoma specimens. Further consequences revealed that ciRS-7 promoted a more aggressive oncogenic phenotype by blocking the miR-7mediated PTEN/PI3K/AKT signaling pathway [50]. Weng et al. revealed that ciRS-7 was significantly elevated in CRC. Significantly higher ciRS-7 levels were found to be related 
to lymph node involvement, more advanced II to IV stages and distant metastasis. In addition, ciRS-7 is an independent risk factor for OS. Mechanistically, ciRS-7 can regulate the EGFR/RAF1/MAPK signaling pathway by competing for miR-7 in CRC [51]. In another study, circRNA-MYLK was significantly upregulated in bladder cancer (BC). Importantly, circRNA-MYLK levels were related to the progression of the stage and grade of $\mathrm{BC}$. Mechanistically, circRNA-MYLK might function as ceRNA for miR-29a, which could contribute to the epithelial-mesenchymal transition and the development of $\mathrm{BC}$ through activating VEGFA/VEGFR2 and the downstream Ras/ERK signaling pathway. These data suggest that circRNA-MYLK would be a promising target for $\mathrm{BC}$ therapy [52]. Another example is circITCH, which positively regulates ITCH by sponging miR-7. ITCH can inhibit the Wnt/B-catenin pathway and thus circITCH can suppress lung cancer, oesophageal squamous cancer and CRC from progression partially via inhibiting the Wnt/ $\beta$-catenin pathway [53-55]. Therefore, circularization may be the future target of cancer treatment.

\section{CircRNAs as potential therapeutic vectors}

The superior ability of circRNA in sponging proteins and miRNAs and its unique cellular stability make it a promising therapeutic vector. Cancer may be stopped by restoring the normal regulatory network through exogenous introduction of circRNA which has multiple binding sites for oncogenic proteins and/or miRNAs [56, 57]. The sponging repertoire can include combinations of miRNA and protein binding sites specifically designed to target specific oncogenic profiles. Furthermore, as the expression of circRNA is regulated by Pol II promoters, we can use disease-activated control elements to limit circRNA expression to malignant cells or use cellspecific promoters to control circRNA expression to certain cell types [19]. Herein, circRNA is a promising vector for cancer therapy.

\section{Therapeutic strategies targeting circRNAs Antagonizing circRNA function \\ Antagonizing circRNAs by short interfering RNAs}

A viable approach to silence circRNA might be to target its unique back-splice junction by exogenously delivering a short interfering RNA (siRNA) perfectly complementary to this site (Fig. 1a). It is important to note that using siRNA to antagonize circRNA should be carried out in a manner that does not interfere with linear mRNA expression [58]. In recent years, siRNA has been applied to effectively silence circRNA in in vivo and in vitro studies [59-61]. However, the offtarget effect is a most noteworthy problem that needs to be carefully avoided [62].

\section{Antagonizing circRNAs by anti-sense oligonucleotides}

Anti-sense oligonucleotides (ASOs), which are shortstranded single sequences, are used as an alternative to target the primary structure of circRNAs (Fig. 1b). It is feasible to knockdown ElciRNA or ciRNA using ASOs targeting intron sequences or back-splicing junction. For instances, Zhang et al. can efficiently knock down ciRNA with appropriate ASO targeting intron sequence [9]. Meanwhile, Li et al. successfully designed ASOs complementary to intron sequences or to the back-splicing junction in the EIciRNA [10]. A possible alternative approach to silence circRNA by exogenous ASOs is to interfere with the back-splicing signals in the pre-mRNA, such as the binding sites for transacting splicing factors or the flanking intronic Alu repeats [19]. Additionally, ASOs should be designed against circRNAs carefully. Otherwise, off-target effects of cognate parent gene suppression might be incurred [63].

\section{Antagonizing circRNAs by CRISPER/Cas system}

Clustered regularly interspaced short palindromic repeatsassociated nuclease Cas9 (CRISPR/Cas) can function quickly and efficiently to cause circRNA to be partially or totally deleted without impeding the linear mRNA based on the deletion of one pair of a flanking intronic reverse complementary match (Fig. 1c). For example, in vitro, when the intronic RNA pairing across the circle-forming exons was disrupted by CRISPR-Cas9-mediated genome editing at the examined circRNA-producing locus, circGCN1L1 could not be detected at this locus at all [64]. Another successful example was circHIPK3, which was demonstrated by Zheng et al. [65]. Additionally, Rajewsky et al. successfully created an in vivo loss-of-function model for Cdrlas from the mouse genome using CRISPR/Cas9 with Cas9 mRNA and two sgRNAs designed to bind upstream of Cdrlas splice sites [66]. Herein, CRISPR/Cas9 technology offers an unprecedented opportunity for partial or complete removal of oncogenic circRNA.

\section{Antagonizing circRNAs by blocking molecular interactions}

When the molecular interactions of circRNAs are blocked, they will be impaired. When small-molecule inhibitors shield the binding points in the partners of protein interaction binding to circRNAs, the above description can be realized. There is another basic principle of the method, that is, to block the combination between circRNA and miRNA by making the conserved binding sites on circRNA saturated (Fig. 1d). Additionally, this is expected to provide a new viewpoint for cancer therapy $[67,68]$.

\section{Restoring circRNA function}

When circRNA acts as a sponge for RNA-binding proteins (RBPs) or miRNAs, reintroduction of circRNA may restore controlled proliferation of the cancer cells or induce apoptosis (Fig. 1e). To date, several studies take 


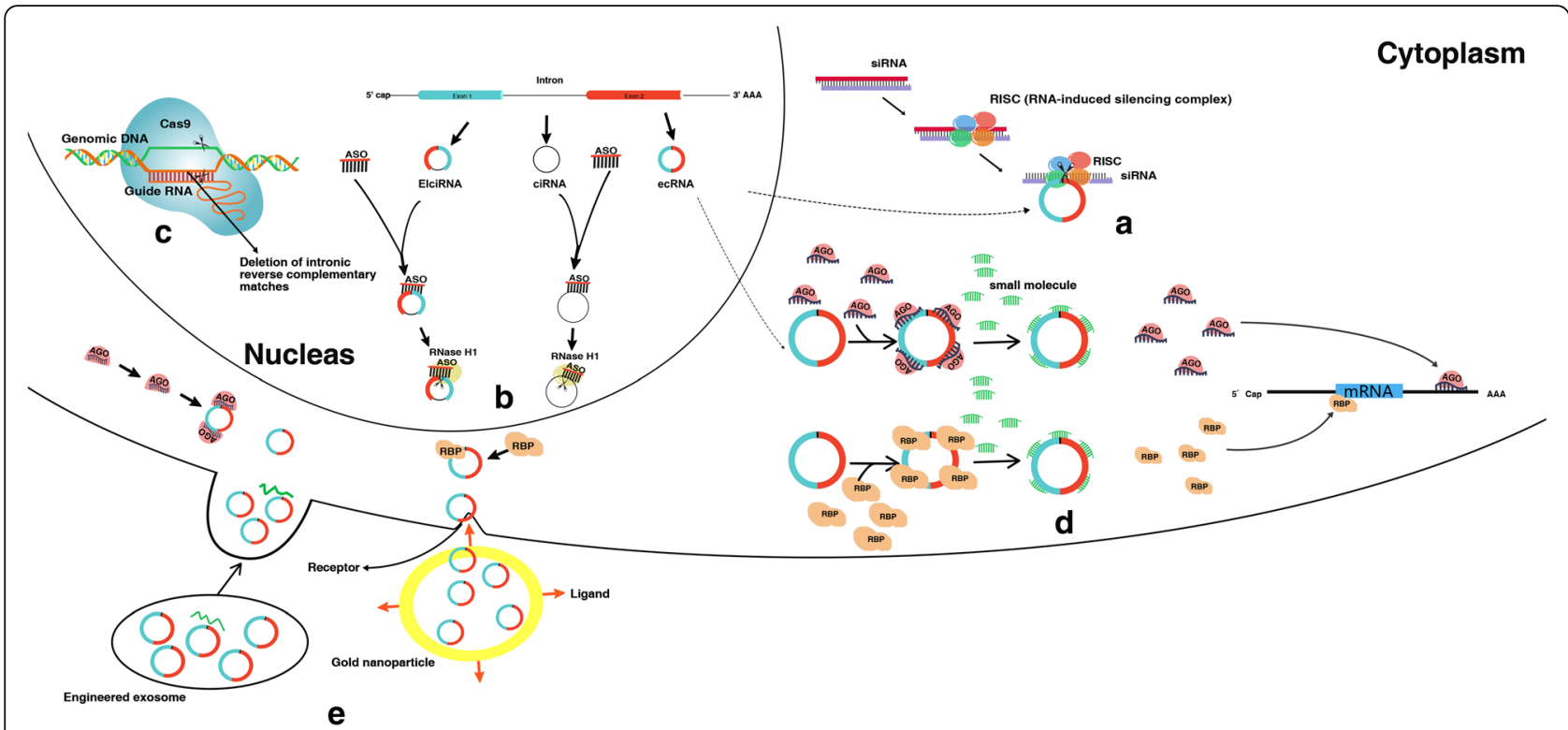

Fig. 1 Possible approaches to target circRNAs. a siRNA-mediated circRNA-specific silencing. b ASO-mediated circRNA-specific silencing. c knockdown or knockout circRNA using CRISPR/Cas9. d Blockade of the combination between circRNA and miRNA or RBP by saturating the binding sites conserved on circRNA. e Reintroduction of circRNAs that are miRNA or RBP sponges

advantage of a minigene construct containing only the exon to be circularized and parts of the flanking introns to express the circRNA of interest $[5,21,69,70]$. However, although often used as a very convenient tool that allows overexpression of circRNAs in vitro, additional linear and circRNA products are often produced $[69,70]$. Hence, to further research circRNAs, minigene construct design should be improved and alternative methods should be developed to facilitate circRNA overexpression [71].

\section{Delivery of circRNAs}

Extracellular vesicles (EVs) are small lipid bilayer vesicles released by cells [72], which can be retooled as delivery vectors for therapeutic intervention [73, 74]. Recently, circRNAs were found to be both enriched and stable in the EVs of cancer cells [35, 75, 76]. In addition, circRNAs in cancer cells can be delivered to exosomes [77]. Furthermore, cells may use EVs to transport circRNAs to communicate to other cells [4]. Therefore, it is possible that EVs, including exosomes and microvesicles, could be engineered to deliver circRNAs efficiently to a target tissue $[48,75]$.

Nanoparticles have emerged as another promising approach for the delivery of circRNAs. Wu et al. has shown that, in vivo, the circular RNA circ-Foxo3 can be delivered to inhibit tumor progression by conjugating the expression plasmids of circ-Foxo3 with gold nanoparticles [78]. Since nanoparticles are not able to enter the nucleus, the therapeutic approach can only focus on exonic circRNAs, which are mainly detected in the cytoplasm.

\section{Conclusions}

The powerful functions and unique properties of circRNAs have made them the focus of scientific and clinical research. In this review, we summarize the recent findings on circRNAs and highlight their clinical value in cancer. First, circRNAs could act as promising biomarkers in cancer diagnosis, prognosis, recurrence, risk evaluation, and response to chemoradiotherapy. Second, circRNAs are potential therapeutic targets and promising vehicles for the delivery of therapeutics. Engineered circRNAs might be effective for sequestering oncogenic miRNAs and RBPs. Third, extracellular vesicles and nanoparticles have emerged as a promising approach for the delivery of circRNAs. In conclusion, circRNAs provide a new perspective for the diagnosis and treatment of cancer.

\section{Perspective}

CircRNAs provide new insights into the "dark matter" of the human genome [79]. However, the field of circRNAs remains largely unexplored and much progress has to be made to incorporate circRNAs into clinical practice.

First, more controlled clinical studies comprising a large number of patients are required before cancerspecific circRNAs can be recommended for human use.

Second, more detailed studies on how to deliver circRNAs efficiently to the accurate action site with a long-term sustained effect and without immunological rejection are needed.

Third, the precise mechanisms of circRNA underlying the initiation and progression of cancer are worth an indepth and extensive study, which is of significance for 
individual therapeutic regimens. Here are some recommendations for future research of circRNAs in cancer.

For the discovery of novel circRNA species involved in cancer, numerous algorithms have been used to detect genome-wide circRNA expression from RNA-seq data in the past few years, but there is little overlap in their predictions [12]. Herein, it is advisable to run more than one circRNA prediction algorithm to minimize the number of false positives in the absence of a gold standard algorithm.

For the stringent circRNA validation method, northern blotting is a more accurate way to fully characterize RNA species than qRT-PCR as no reverse transcription and amplification steps are part of the protocol [80]. Fluorescence in situ hybridization (FISH) provides spatial information about specific circRNAs, which is likely to be employed more in future circRNA cancer research [81].

To discover the detail binding sites for circRNA-miRNA and circRNA-RBP interactions, AGO2 RNA immunoprecipitation (RIP) combined with luciferase reporter assay, RIP followed by sequencing, and RNA pull down combined with mass spectrometry are suggested methods [82, 83].

To elucidate circRNA cellular roles, it is indispensable that exogenous synthetic molecules with engineered sequences should be designed to mimic or antagonize circRNAs. Furthermore, these molecules should be able to decoy or release miRNAs and RBPs. This research method is important to obtain circRNAs' accurate epigenetic roles and corresponding mechanisms in cancer.

Dynamic analysis of aberrantly expressed circRNAs in cancer, such as in different stages or different sensitivities to a specific chemotherapy treatment, is very crucial for further understanding and clarifying the mechanism underlying cancer development and presenting potential therapeutic targets. A preliminary study found a large number of differentially expressed circRNAs between primary ovarian tumor and metastases using RNA-seq, which could offer a more robust classifier, for tumor subtypes or provide biomarkers for tumor screening and prognosis [84]. Another preliminary study on chemoresistant breast cancer provided several appealing targets for further functional analysis according to circRNA microarray data [47]. More comprehensive studies on those differentially expressed circRNAs will certainly gain more insights of the expression dynamics and functions of circRNAs in cancer.

We hope that one day, the appropriate and precise use of circRNAs in clinical diagnosis and treatment will become a new foothold for translational and precision medicine.

\footnotetext{
Abbreviations

5-FU: 5-Fluorouracil; ADM: Adriamycin; AGO: Argonaute; ASO: Anti-sense oligonucleotide; BC: Bladder cancer; ceRNA: Competing endogenous RNA; circRNAs: Circular RNAs; ciRNA: Intronic circRNA; ciRS-7: Circular RNA sponge for miR-7; CRC: Colorectal cancer; CRISPR/Cas9: Clustered regularly interspaced short palindromic repeats-associated nuclease Cas9; ecRNA: Exonic circRNA; ElciRNA: Exon-intron circRNA; EV: Extracellular vesicle; FISH: Fluorescence in situ hybridization; GC: Gastric cancer; HCC: Hepatocellular cancer; IRES: Internal
}

ribosome entry site; LAC: Lung adenocarcinoma; m6A: $N^{6}$-Methyladenosine; miRNA: MicroRNA; OS: Overall survival; PFS: Progression-free survival; Pol II: RNA polymerase II; qRT-PCR: Quantitative real-time PCR; RBP: RNA-binding protein; RCA: Rolling circle amplification; RIP: RNA immunoprecipitation; RNA-seq: RNA sequencing; ROC: Receiver operating characteristic; SiRNA: Short interfering RNA; SNP: Single nucleotide polymorphism; snRNA: U1 small nuclear RNA; snRNP: Small nuclear ribonucleoprotein; SRY: Sex-determining region Y; TNM: Tumor-node-metastasis

\section{Acknowledgements}

Not applicable.

\section{Funding}

This work was supported by the National Natural Science Foundation of China (No. 81071650), the Special foundation for Science and Technology Program in Liaoning Province, China (2013225585), and the Supporting Project for Climbing Scholars in Liaoning Provincial Universities, China (2009).

Availability of data and materials Not applicable.

Authors' contributions

All authors have contributed to the preparation of this manuscript. Both authors read and approved the final manuscript.

Ethics approval and consent to participate

Not applicable.

\section{Consent for publication}

Not applicable.

\section{Competing interests}

The authors declare that they have no competing interests.

\section{Publisher's Note}

Springer Nature remains neutral with regard to jurisdictional claims in published maps and institutional affiliations.

Received: 20 December 2017 Accepted: 6 February 2018 Published online: 13 February 2018

\section{References}

1. Vitiello M, Tuccoli A, Poliseno L. Long non-coding RNAs in cancer: implications for personalized therapy. Cell Oncol (Dordr). 2015;38(1):17-28.

2. Huang G, Li S, Yang N, Zou Y, Zheng D, Xiao T. Recent progress in circular RNAs in human cancers. Cancer Lett. 2017;404:8-18.

3. Qu S, Liu Z, Yang $X$, Zhou J, Yu H, Zhang R, et al. The emerging functions and roles of circular RNAs in cancer. Cancer Lett. 2018;414:301-9.

4. Greene J, Baird AM, Brady L, Lim M, Gray SG, McDermott R, et al. Circular RNAs: biogenesis, function and role in human diseases. Front Mol Biosci. 2017:4:38.

5. Ashwal-Fluss R, Meyer M, Pamudurti NR, Ivanov A, Bartok O, Hanan M, et al. CircRNA biogenesis competes with pre-mRNA splicing. Mol Cell. 2014;56(1):55-66.

6. Starke $\mathrm{S}$, Jost I, Rossbach $\mathrm{O}$, Schneider T, Schreiner $\mathrm{S}$, Hung LH, et al. Exon circularization requires canonical splice signals. Cell Rep. 2015;10(1):103-11.

7. Zhu LP, He YJ, Hou JC, Chen X, Zhou SY, Yang SJ, et al. The role of circRNAs in cancers. Biosci Rep. 2017:37(5):e20170750.

8. Zhang XO, Wang HB, Zhang Y, Lu X, Chen LL, Yang L. Complementary sequence-mediated exon circularization. Cell. 2014;159(1):134-47.

9. Zhang $Y$, Zhang $X O$, Chen $T$, Xiang JF, Yin QF, Xing YH, et al. Circular intronic long noncoding RNAs. Mol Cell. 2013;51:792-806.

10. Li Z, Huang C, Bao C, Chen L, Lin M, Wang $X$, et al. Exon-intron circular RNAs regulate transcription in the nucleus. Nat Struct Mol Biol. 2015; 22(3):256-64

11. Vidal AF, Sandoval GT, Magalhães L, Santos SE, Ribeiro-dos-Santos Â. Circular RNAs as a new field in gene regulation and their implications in translational research. Epigenomics. 2016;8(4):551-62.

12. Szabo L, Salzman J. Detecting circular RNAs: bioinformatic and experimental challenges. Nat Rev Genet. 2016;17(11):679-92. 
13. Memczak S, Papavasileiou P, Peters O, Rajewsky N. Identification and characterization of circular RNAs as a new class of putative biomarkers in human blood. PLoS One. 2015;10(10):e0141214.

14. Ivanov A, Memczak S, Wyler E, Torti F, Porath HT, Orejuela MR, et al. Analysis of intron sequences reveals hallmarks of circular RNA biogenesis in animals. Cell Rep. 2015;10(2):170-7.

15. Conn SJ, Pillman KA, Toubia J, Conn VM, Salmanidis M, Phillips CA, et al. The RNA-binding protein quaking regulates formation of circRNAs. Cell. 2015; 160(6):1125-34.

16. Dong $Y$, He D, Peng Z, Peng W, Shi W, Wang J, et al. Circular RNAs in cancer: an emerging key player. J Hematol Oncol. 2017;10(1):2.

17. Chen X, Fan S, Song E. Noncoding RNAs: new players in cancers. Adv Exp Med Biol. 2016:927:1-47.

18. Han YN, Xia SQ, Zhang YY, Zheng JH, Li W. Circular RNAs: a novel type of biomarker and genetic tools in cancer. Oncotarget. 2017:8(38):64551-63.

19. Kristensen LS, Hansen TB, Venø MT, Kjems J. Circular RNAs in cancer: opportunities and challenges in the field. Oncogene. 2017; https://doi.org/10.1038/onc.2017.361.

20. Rong D, Tang W, Li Z, Zhou J, Shi J, Wang H, et al. Novel insights into circular RNAs in clinical application of carcinomas. Onco Targets Ther. 2017; 10:2183-8

21. Hansen TB, Jensen TI, Clausen BH, Bramsen JB, Finsen B, Damgaard CK, et al. Natural RNA circles function as efficient microRNA sponges. Nature. 2013; 495(7441):384-8

22. Memczak S, Jens M, Elefsinioti A, Torti F, Krueger J, Rybak A, et al. Circular RNAs are a large class of animal RNAs with regulatory potency. Nature. 2013:495(7441):333-8.

23. Capel B, Swain A, Nicolis S, Hacker A, Walter M, Koopman P, et al. Circular transcripts of the testis-determining gene Sry in adult mouse testis. Cell. 1993;73(5):1019-30.

24. Jeck WR, Sharpless NE. Detecting and characterizing circular RNAs. Nat Biotechnol. 2014;32(5):453-61

25. Du WW, Yang W, Liu E, Yang Z, Dhaliwal P, Yang BB. Foxo3 circular RNA retards cell cycle progression via forming ternary complexes with p21 and CDK2. Nucleic Acids Res. 2016;44(6):2846-58.

26. Wang $Y$, Wang Z. Efficient backsplicing produces translatable circular mRNAs. RNA. 2015:21(2):172-9.

27. Chen CY, Sarnow P. Initiation of protein synthesis by the eukaryotic translational apparatus on circular RNAs. Science. 1995:268(5209):415-7.

28. Abe N, Matsumoto K, Nishihara M, Nakano Y, Shibata A, Maruyama H, et al. Rolling circle translation of circular RNA in living human cells. Sci Rep. 2015; 5:16435.

29. Weingarten-Gabbay S, Elias-Kirma S, Nir R, Gritsenko AA, Stern-Ginossar N, Yakhini Z, et al. Comparative genetics. Systematic discovery of capindependent translation sequences in human and viral genomes. Science. 2016:351:6270

30. Pamudurti NR, Bartok O, Jens M, Ashwal-Fluss R, Stottmeister C, Ruhe L, et al. Translation of CircRNAs. Mol Cell. 2017;66(1):9-21.

31. Legnini I, Di Timoteo G, Rossi F, Morlando M, Briganti F, Sthandier O, et al. Circ-ZNF609 is a circular RNA that can be translated and functions in myogenesis. Mol Cell. 2017;66(1):22-37.

32. Yang $Y$, Fan $X$, Mao $M$, Song $X$, Wu $P$, Zhang $Y$, et al. Extensive translation of circular RNAs driven by $N^{6}$-methyladenosine. Cell Res. 2017;27(5):626-41.

33. Wang $Y$, Mo Y, Gong Z, Yang X, Yang M, Zhang S, et al. Circular RNAs in human cancer. Mol Cancer. 2017;16(1):25.

34. Zhu X, Wang X, Wei S, Chen Y, Chen Y, Fan X, et al. hsa circ 0013958: a circular RNA and potential novel biomarker for lung adenocarcinoma. FEBS J. 2017;284(14):2170-82

35. Li Y, Zheng Q, Bao C, Li S, Guo W, Zhao J, et al. Circular RNA is enriched and stable in exosomes: a promising biomarker for cancer diagnosis. Cell Res. 2015;25(8):981-4.

36. Benna C, Helfrich-Förster C, Rajendran S, Monticelli H, Pilati P, Nitti D, et al. Genetic variation of clock genes and cancer risk: a field synopsis and metaanalysis. Oncotarget. 2017:8(14):23978-95.

37. Guo W, Zhang J, Zhang D, Cao S, Li G, Zhang S, et al. Polymorphisms and expression pattern of circular RNA circ-ITCH contributes to the carcinogenesis of hepatocellular carcinoma. Oncotarget. 2017;8(29):48169-77.

38. Yang Y, Gao X, Zhang M, Yan S, Sun C, Xiao F, et al. Novel role of FBXW7 circular RNA in repressing glioma tumorigenesis. J Natl Cancer Inst. 2018;110:3.

39. Zhang H, Wang G, Ding C, Liu P, Wang R, Ding W, et al. Increased circular RNA UBAP2 acts as a sponge of miR-143 to promote osteosarcoma progression. Oncotarget. 2017;8(37):61687-97.
40. Yao JT, Zhao SH, Liu QP, Lv MQ, Zhou DX, Liao ZJ, et al. Over-expression of CircRNA 100876 in non-small cell lung cancer and its prognostic value. Pathol Res Pract. 2017;213(5):453-6.

41. Fang $Y$, Ma M, Wang J, Liu X, Wang Y. Circular RNAs play an important role in late-stage gastric cancer: circular RNA expression profiles and bioinformatics analyses. Tumour Biol. 2017;39(6):1010428317705850.

42. Chen J, Li Y, Zheng Q, Bao C, He J, Chen B, et al. Circular RNA profile identifies circPVT1 as a proliferative factor and prognostic marker in gastric cancer. Cancer Lett. 2017;388:208-19.

43. Zhu J, Ye J, Zhang L, Xia L, Hu H, Jiang H, et al. Differential expression of circular RNAs in glioblastoma multiforme and its correlation with prognosis. Transl Oncol. 2017;10(2):271-9.

44. Su H, Lin F, Deng $X$, Shen L, Fang $Y$, Fei $Z$, et al. Profiling and bioinformatics analyses reveal differential circular RNA expression in radioresistant esophageal cancer cells. J Transl Med. 2016;14(1):225.

45. Guarnerio J, Bezzi M, Jeong JC, Paffenholz SV, Berry K, Naldini MM, et al. Oncogenic role of fusion-circRNAs derived from cancer-associated chromosomal translocations. Cell. 2016;166(4):1055-6.

46. Xiong W, Ai YQ, Li YF, Ye Q, Chen ZT, Qin JY, et al. Microarray analysis of circular RNA expression profile associated with 5-fluorouracil-based chemoradiation resistance in colorectal cancer cells. Biomed Res Int. 2017: 2017:8421614.

47. Gao D, Zhang X, Liu B, Meng D, Fang K, Guo Z, et al. Screening circular RNA related to chemotherapeutic resistance in breast cancer. Epigenomics. 2017; 9(9):1175-88.

48. Liu J, Liu T, Wang X, He A. Circles reshaping the RNA world: from waste to treasure. Mol Cancer. 2017;16(1):58.

49. Yang Z, Xie L, Han L, Qu X, Yang Y, Zhang Y, et al. Circular RNAs: regulators of cancer-related signaling pathways and potential diagnostic biomarkers for human cancers. Theranostics. 2017:7(12):3106-17.

50. Pan H, Li T, Jiang Y, Pan C, Ding Y, Huang Z, et al. Overexpression of circular RNA ciRS-7 abrogates the tumor suppressive effect of miR-7 on gastric cancer via PTEN/PI3K/AKT signaling pathway. J Cell Biochem. 2018;119(1):440-6.

51. Weng W, Wei Q, Toden S, Yoshida K, Nagasaka T, Fujiwara T, et al. Circular RNA ciRS-7-a promising prognostic biomarker and a potential therapeutic target in colorectal cancer. Clin Cancer Res. 2017;23(14):3918-28.

52. Zhong Z, Huang M, Lv M, He Y, Duan C, Zhang L, et al. Circular RNA MYLK as a competing endogenous RNA promotes bladder cancer progression through modulating VEGFANEGFR2 signaling pathway. Cancer Lett. 2017;403:305-17.

53. Wan L, Zhang L, Fan K, Cheng ZX, Sun QC, Wang JJ. Circular RNA-ITCH suppresses lung cancer proliferation via inhibiting the Wnt/ $\beta$-catenin pathway. Biomed Res Int. 2016;2016:1579490.

54. Li F, Zhang L, Li W, Deng J, Zheng J, An M, et al. Circular RNA ITCH has inhibitory effect on ESCC by suppressing the Wnt/ $\beta$-catenin pathway. Oncotarget. 2015;6(8):6001-13.

55. Huang G, Zhu H, Shi Y, Wu W, Cai H, Chen X. Cir-ITCH plays an inhibitory role in colorectal cancer by regulating the $\mathrm{Wnt} / \mathrm{\beta}$-catenin pathway. PLoS One. 2015;10(6):e0131225.

56. Bachmayr-Heyda A, Auer K, Sukhbaatar N, Aust S, Deycmar S, Reiner AT, et al. Small RNAs and the competing endogenous RNA network in high grade serous ovarian cancer tumor spread. Oncotarget. 2016;7(26):39640-53.

57. Huang M, Zhong Z, Lv M, Shu J, Tian Q, Chen J. Comprehensive analysis of differentially expressed profiles of IncRNAs and circRNAs with associated coexpression and ceRNA networks in bladder carcinoma. Oncotarget. 2016; 7(30):47186-200

58. Cortés-López M, Miura P. Emerging functions of circular RNAs. Yale J Biol Med. 2016:89(4):527-37.

59. Hsiao KY, Lin YC, Gupta SK, Chang N, Yen L, Sun HS, et al. Noncoding effects of circular RNA CCDC66 promote colon cancer growth and metastasis. Cancer Res. 2017:77(9):2339-50.

60. Yao Z, Luo J, Hu K, Lin J, Huang H, Wang Q, et al. ZKSCAN1 gene and its related circular RNA (circZKSCAN1) both inhibit hepatocellular carcinoma cell growth, migration, and invasion but through different signaling pathways. Mol Oncol. 2017:11(4):422-37.

61. Li P, Chen H, Chen S, Mo X, Li T, Xiao B, et al. Circular RNA 0000096 affects cell growth and migration in gastric cancer. Br J Cancer. 2017:116(5):626-33.

62. Wang T, Shigdar S, Shamaileh HA, Gantier MP, Yin W, Xiang D, et al. Challenges and opportunities for siRNA-based cancer treatment. Cancer Lett. 2017;387:77-83.

63. Frazier KS. Antisense oligonucleotide therapies: the promise and the challenges from a toxicologic pathologist's perspective. Toxicol Pathol. 2015; 43(1):78-89. 
64. Zhang Y, Xue W, Li X, Zhang J, Chen S, Zhang JL, et al. The biogenesis of nascent circular RNAs. Cell Rep. 2016;15(3):611-24.

65. Zheng Q, Bao C, Guo W, Li S, Chen J, Chen B, et al. Circular RNA profiling reveals an abundant circHIPK3 that regulates cell growth by sponging multiple miRNAs. Nat Commun. 2016;7:11215.

66. Piwecka M, Glažar P, Hernandez-Miranda LR, Memczak S, Wolf SA, RybakWolf $A$, et al. Loss of a mammalian circular RNA locus causes miRNA deregulation and affects brain function. Science. 2017;357(6357):eaam8526

67. Tay FC, Lim JK, Zhu H, Hin LC, Wang S. Using artificial microRNA sponges to achieve microRNA loss-of-function in cancer cells. Adv Drug Deliv Rev. 2015; 81:117-27.

68. Zhao ZJ, Shen J. Circular RNA participates in the carcinogenesis and the malignant behavior of cancer. RNA Biol. 2017;14(5):514-21.

69. Liang D, Wilusz JE. Short intronic repeat sequences facilitate circular RNA production. Genes Dev. 2014;28(20):2233-47.

70. Barrett SP, Wang PL, Salzman J. Circular RNA biogenesis can proceed through an exon-containing lariat precursor. elife. 2015:4:e07540.

71. Petkovic S, Müller S. RNA circularization strategies in vivo and in vitro. Nucleic Acids Res. 2015;43(4):2454-65.

72. Ma P, Pan Y, Li W, Sun C, Liu J, Xu T, et al. Extracellular vesicles-mediated noncoding RNAs transfer in cancer. J Hematol Oncol. 2017;10(1):57.

73. Tian Y, Li S, Song J, Ji T, Zhu M, Anderson GJ, et al. A doxorubicin delivery platform using engineered natural membrane vesicle exosomes for targeted tumor therapy. Biomaterials. 2014;35(7):2383-90.

74. Yang T, Martin P, Fogarty B, Brown A, Schurman K, Phipps R, et al. Exosome delivered anticancer drugs across the blood-brain barrier for brain cancer therapy in Danio rerio. Pharm Res. 2015;32(6):2003-14.

75. Lasda E, Parker R. Circular RNAs co-precipitate with extracellular vesicles: a possible mechanism for circRNA clearance. PLoS One. 2016;11(2):e0148407.

76. Kim KM, Abdelmohsen K, Mustapic M, Kapogiannis D, Gorospe M. RNA in extracellular vesicles. Wiley Interdiscip Rev RNA. 2017;8(4):e1413.

77. Dou Y, Cha DJ, Franklin JL, Higginbotham JN, Jeppesen DK, Weaver AM, et al. Circular RNAs are down-regulated in KRAS mutant colon cancer cells and can be transferred to exosomes. Sci Rep. 2016;6:37982.

78. Du WW, Fang L, Yang W, Wu N, Awan FM, Yang Z, et al. Induction of tumor apoptosis through a circular RNA enhancing Foxo3 activity. Cell Death Differ. 2017;24(2):357-70.

79. Chen Y, Li C, Tan C, Liu X. Circular RNAs: a new frontier in the study of human diseases. J Med Genet. 2016;53(6):359-65.

80. Patop IL, Kadener S. CircRNAs in cancer. Curr Opin Genet Dev. 2017:48:121-7.

81. Zirkel A, Papantonis A. Detecting circular RNAs by RNA fluorescence in situ hybridization. Methods Mol Biol. 2018;1724:69-75.

82. Li Y, Chen B, Huang S. Identification of circRNAs for miRNA targets by Argonaute2 RNA immunoprecipitation and luciferase screening assays. Methods Mol Biol. 2018;1724:209-18.

83. Du WW, Zhang C, Yang W, Yong T, Awan FM, Yang BB. Identifying and characterizing circRNA-protein interaction. Theranostics. 2017;7(17):4183-91.

84. Ahmed I, Karedath T, Andrews SS, Al-Azwani IK, Mohamoud YA, Querleu D, et al. Altered expression pattern of circular RNAs in primary and metastatic sites of epithelial ovarian carcinoma. Oncotarget. 2016;7(24):36366-81.

85. Zhong Z, Lv M, Chen J. Screening differential circular RNA expression profiles reveals the regulatory role of circTCF25-miR-103a-3p/miR-107-CDK6 pathway in bladder carcinoma. Sci Rep. 2016;6:30919.

86. Kong Z, Wan X, Zhang Y, Zhang P, Zhang Y, Zhang X, et al. Androgen-responsive circular RNA circSMARCA5 is up-regulated and promotes cell proliferation in prostate cancer. Biochem Biophys Res Commun. 2017;493(3):1217-23.

87. Zheng J, Liu X, Xue Y, Gong W, Ma J, Xi Z, et al. TTBK2 circular RNA promotes glioma malignancy by regulating miR-217/HNF1 $\beta /$ Derlin-1 pathway. J Hematol Oncol. 2017;10(1):52.

88. Li JF, Song YZ. Circular RNA hsa_circ_0001564 facilitates tumorigenesis of osteosarcoma via sponging miR-29c-3p. Tumour Biol. 2017;39(8):10.1177/ 1010428317709989

89. Tang YY, Zhao P, Zou TN, Duan JJ, Zhi R, Yang SY, et al. Circular RNA hsa_ circ_0001982 promotes breast cancer cell carcinogenesis through decreasing miR-143. DNA Cell Biol. 2017;36(11):901-8.

90. Lü L, Sun J, Shi P, Kong W, Xu K, He B, et al. Identification of circular RNAs as a promising new class of diagnostic biomarkers for human breast cancer. Oncotarget. 2017;8(27):44096-107.

91. Liang HF, Zhang XZ, Liu BG, Jia GT, Li WL. Circular RNA circ-ABCB10 promotes breast cancer proliferation and progression through sponging miR-1271. Am J Cancer Res. 2017;7(7):1566-76.
92. Yu L, Gong X, Sun L, Zhou Q, Lu B, Zhu L. The circular RNA Cdr1as act as an oncogene in hepatocellular carcinoma through targeting miR-7 expression. PLoS One. 2016;11(7):e0158347.

93. Ren S, Xin Z, Xu Y, Xu J, Wang G. Construction and analysis of circular RNA molecular regulatory networks in liver cancer. Cell Cycle. 2017;16(22):2204-11.

94. Shang X, Li G, Liu H, Li T, Liu J, Zhao Q, et al. Comprehensive circular RNA profiling reveals that hsa_circ_0005075, a new circular RNA biomarker, is involved in hepatocellular carcinoma development. Medicine (Baltimore). 2016;95(22):e3811.

95. Xia W, Qiu M, Chen R, Wang S, Leng X, Wang J, et al. Circular RNA has_circ_ 0067934 is upregulated in esophageal squamous cell carcinoma and promoted proliferation. Sci Rep. 2016;6:35576.

96. Zhu M, Xu Y, Chen Y, Yan F. Circular BANP, an upregulated circular RNA that modulates cell proliferation in colorectal cancer. Biomed Pharmacother. 2017:88:138-44.

97. Zhang XL, Xu LL, Wang F. Hsa_circ_0020397 regulates colorectal cancer cell viability, apoptosis and invasion by promoting the expression of the miR138 targets TERT and PD-L1. Cell Biol Int. 2017;41(9):1056-64.

98. Xuan L, Qu L, Zhou H, Wang P, Yu H, Wu T, et al. Circular RNA: a novel biomarker for progressive laryngeal cancer. Am J Transl Res. 2016;8(2):932-9.

99. Li Y, Zheng F, Xiao X, Xie F, Tao D, Huang C, et al. CircHIPK3 sponges miR558 to suppress heparanase expression in bladder cancer cells. EMBO Rep. 2017;18(9):1646-59.

100. Xie H, Ren X, Xin S, Lan X, Lu G, Lin Y, et al. Emerging roles of circRNA 001569 targeting miR-145 in the proliferation and invasion of colorectal cancer. Oncotarget. 2016;7(18):26680-91.

101. Zhang P, Zuo Z, Shang W, Wu A, Bi R, Wu J, et al. Identification of differentially expressed circular RNAs in human colorectal cancer. Tumour Biol. 2017;39(3):1010428317694546.

102. Fu L, Wu S, Yao T, Chen Q, Xie Y, Ying S, et al. Decreased expression of hsa circ_0003570 in hepatocellular carcinoma and its clinical significance. J Clin Lab Anal. 2017; https://doi.org/10.1002/jcla.22239.

103. Han D, Li J, Wang H, Su X, Hou J, Gu Y, et al. Circular RNA circMTO1 acts as the sponge of microRNA-9 to suppress hepatocellular carcinoma progression. Hepatology. 2017;66(4):1151-64.

104. Huang XY, Huang ZL, Xu YH, Zheng Q, Chen Z, Song W, et al. Comprehensive circular RNA profiling reveals the regulatory role of the circRNA-100338/miR-141-3p pathway in hepatitis B-related hepatocellular carcinoma. Sci Rep. 2017;7(1):5428.

105. Li J, Zhen L, Zhang Y, Zhao L, Liu H, Cai D, et al. Circ-104916 is downregulated in gastric cancer and suppresses migration and invasion of gastric cancer cells. Onco Targets Ther. 2017;10:3521-9.

106. Lu R, Shao Y, Ye G, Xiao B, Guo J. Low expression of hsa_circ_0006633 in human gastric cancer and its clinical significances. Tumour Biol. 2017;39(6): 1010428317704175.

107. Zhao Q, Chen S, Li T, Xiao B, Zhang X. Clinical values of circular RNA 0000181 in the screening of gastric cancer. J Clin Lab Anal. 2017; https://doi.org/10.1002/jcla.22333.

108. Zhang Y, Liu H, Li W, Yu J, Li J, Shen Z, et al. CircRNA_100269 is downregulated in gastric cancer and suppresses tumor cell growth by targeting miR-630. Aging (Albany NY). 2017;9(6):1585-94.

109. Li P, Chen S, Chen H, Mo X, Li T, Shao Y, et al. Using circular RNA as a novel type of biomarker in the screening of gastric cancer. Clin Chim Acta. 2015; 444:132-6.

110. Chen S, Li T, Zhao Q, Xiao B, Guo J. Using circular RNA hsa_circ_0000190 as a new biomarker in the diagnosis of gastric cancer. Clin Chim Acta. 2017; 466:167-71.

111. Shao Y, Li J, Lu R, Li T, Yang Y, Xiao B, et al. Global circular RNA expression profile of human gastric cancer and its clinical significance. Cancer Med. 2017;6(6):1173-80

112. Tian M, Chen R, Li T, Xiao B. Reduced expression of circRNA hsa_circ 0003159 in gastric cancer and its clinical significance. J Clin Lab Anal. 2017; https://doi.org/10.1002/jcla.22281. 